\title{
Atorvastatin prescription before and after Percutaneous Coronary Intervention: Effects on Left Ventricular Ejection Fraction and blood circulating Pentraxin3
}

\author{
Namazi $\mathrm{MH}^{1}$, Alipourparsa $\mathrm{S}^{1}$, Kiani $\mathrm{Y}^{2}$, Meamaryan $\mathrm{M}^{1}$, Khaheshi $\mathrm{I}^{1}$ and Haybar $\mathrm{H}^{{ }_{3}}$ \\ ${ }^{1}$ Cardiovascular Research Center, Modarres Hospital, Shahid Beheshti University of Medical Sciences, Tehran, Iran \\ ${ }^{2}$ Isfahan University of Medical Sciences, Isfahan, Iran \\ ${ }^{3}$ Ahvaz Jundishapur University of Medical Sciences, Ahvaz, Iran
}

${ }^{*}$ Corresponding author: Haybar H, Ahvaz Jundishapur University of Medical Sciences, Ahvaz, Iran, Tel: +98916 613 1474, E-mail: takamoolsk@yahoo.com

Citation: Namazi MH, Alipourparsa S, Kiani Y, Meamaryan M, Khaheshi I, et al. (2015) Atorvastatin prescription before and after Percutaneous Coronary Intervention: effects on Left Ventricular Ejection Fraction and blood circulating Pentraxin3. J Clin Exp Res Cardiol 2(2): 201. doi: 10.15744/2394-6504.2.201

Received Date: May 04, 2015 Accepted Date: August 18, 2015 Published Date: August 20, 2015

\begin{abstract}
Introduction: Statins can reduce risk of myocardial infarction, stroke and mortality in high risk individuals and improve clinical outcomes in patients undergoing Percutaneous Coronary Intervention (PCI). The major challenge of physicians at the emergency condition is the fact that patients are not stable and cannot take several pills, wholesale.

Methods: This study was carried out on 100 patients who suffered from AMI with the early plan for primary PCI. All patients were divided to two group, randomly; fifty patients received conventional drug including Atorvastatin (80 mg/day), Aspirin (325 mg/day) and Clopidogrel $(600 \mathrm{mg} /$ day $)$ at the time of admitting to the emergency and before primary-PCI; the other 50 patients take same drug regime without Atorvastatin just like the control group, before primary-PCI and a single dose Atorvastatin ( $80 \mathrm{mg} / \mathrm{day})$ after primary PCI. A paired T-test was employed to study the result of PTX3 and LVEF before and after primary PCI among all patients. An independent T-test was also used for statistical analysis of the result of PTX3 value and EF after primary PCI for both drug plan intervention groups.

Results: PTX3 and LVEF values in the group with Atorvastatin pre-treatment changed from $6.07 \pm 2.31 \mathrm{ng} / \mathrm{dL}$ to $4.92 \pm 1.98 \mathrm{ng} / \mathrm{dL}$ and $40.8 \pm 8.88$ to $45 \pm 6.77$, respectively after primary PCI. Both mentioned indexes in the Atorvastatin post-treatment group were also changed from $5.91 \pm 3.24 \mathrm{ng} / \mathrm{dL}$ to $5.37 \pm 3.6$ and $41.2 \pm 8.66$ to $44.9 \pm 7.79$, respectively, after primary PCI. None statistically significant difference were seen in comparison the results of both LVEF (P value $=0.946)$ and PTX3 $(0.445)$ values between two groups.

Conclusion: Our results indicate that Atorvastatin therapy after primary-PCI may has same beneficial effects on Cardiac functions compared with conventional drug regime before PCI.
\end{abstract}

Keywords: PCI; Atorvastatin; Ejection Fraction; Pentraxin3

\section{Introduction}

Coronary Artery Diseases (CAD) is one of the most prevalent causative agents of morbidity and mortality, worldwide [1]. Many studies have been reported the role of statins that not only reduce risk of myocardial infarction, stroke and mortality in high risk individuals but also improve clinical outcomes among patients undergoing Percutaneous Coronary Intervention (PCI) [2] for stable coronary artery diseases and non-ST segment elevation coronary syndrome $[3,4]$ as well as ST segment elevation myocardial infarction [5].

At the time of PCI, Periprocedural myocardial injury is associated with more complication events [6] and therefore reducing the adverse events is one of the main goals. Previous studies showed that pre-treatment with statins before PCI can reduce cardiovascular complications after PCI $[7,8,4]$. Some studies suggest that statin therapy is able to stabilize coronary plague, decrease the chance of thrombus formation and increase endothelial function [9]. However, the major challenge of physicians at the emergency condition is the fact that the patients are not stable and cannot take several pills (Atorvastatin, Aspirin and Clopidogrel), wholesale. In the other hand, although as mentioned above, almost all clinical trials have reported the efficacy of Atorvastatin pre-treatment of patients undergoing PCI but there are only few studies about Atorvastatin post-treatment of patients after PCI [10].

PTX3 is a new interesting inflammatory indicator that has homology in both structure and function with hs-CRP (highly sensitive C Reactive Protein) [11]. Compared with hs-CRP, this indicator has more specificity role in diagnosis of Coronary Artery Diseases $[12,13]$. Indeed, several studies have showed correlation between PTX3 with CAD and Syntax score $[14,15]$. 
Therefore we aimed to survey the effects of Atorvastatin treatment beside Aspirin and Clopidogrel as conventional therapy before primary PCI with a drug plan as prescribing Aspirin and Clopidogrel before PCI and then a single dose of Atorvastatin (80 mg/ day) after primary PCI on Pentraxin3 (PTX3) and Left Ventricular Ejection Fraction (LVEF) in patients who suffer from Acute Myocardial Infarction (AMI) with an early plan for primary PCI.

\section{Methods}

\section{Study population}

This study was carried out on patients who seeking care from a general hospital (Moddarres Hospital), department of Cardiology, during 2013-2014. For describing AMI, patients who had ST Elevation in ECG leads beside positive troponin or acute and typical chest pain were diagnosed as AMI and underwent primary PCI up to 1 hours after admitting to the hospital.

All patients with prinzmetal angina, pulmonary edema, cardiogenic shock, high blood pressure, and previous history of consuming Atorvastatin were excluded from the study.

A total 100 patients who suffered from AMI with the early plan for primary PCI were enrolled in this study. All patients who were included in the study rested for $15 \mathrm{~min}$ in the sitting position and then blood pressure was taken using a standardized mercury sphygmomanometer. A same physician performed primary PCI and a trained interviewer also filled questionnaires consisted of demographic data, smoking status and history of medical situation using face to face model.

\section{Clinical intervention}

when patients enrolled for the study, they were divided to two equal groups using double blind method, randomly; a control group including 50 patients who received conventional drug regime including $80 \mathrm{mg} /$ day Atorvastatin, $325 \mathrm{mg} / \mathrm{day} \mathrm{Aspirin} \mathrm{and} 600 \mathrm{mg} /$ day Clopidogrel at the time of admitting to the emergency, before primary PCI and the other 50 patients take same drug regime without Atorvastatin at the same time. The latter group received Atorvastatin $80 \mathrm{mg}$ /day post-treatment after primary PCI; 24 hours after admitting to the hospital. At the time of enrolling patients in the study and before primary PCI, LVEF and PTX3 levels were assessed. For the second time, evaluation of PTX3 and LVEF was also performed for all enrolled patients 24 hours and 5 days after admitting to the hospital, respectively.

The same technician using commercial Human Pentraxin-3 ELISA kit (CUSABIO BIOTECH CO., Japan) according to the previous study [16] evaluated PTX3 value. Same persons performed all evaluations on PTX3 levels and LVEF.

\section{Statistical Analysis}

Gathered data was analyzed using SPSS version 22 for windows. Descriptive data for continuous variable for normal and nonnormal distribution was presented as Mean (SD) and median (IQR), respectively. Categorically distributed variables were also described as frequency (n,\%). A paired T-test was employed to study the result of PTX3 and LVEF before and after PCI among patients of both groups. An independent T-test was also used for statistical analysis of the result of PTX3 value and LVEF after PCI for both drug plan intervention groups. P-value less than 0.05 considered as significant in all analysis.

\section{Ethical approval}

All patients who were enrolled in this study were aware about the study as well as drug regime changes and all experiments on the patients were performed based on guidelines of the ethical board of Shahid Beheshti University of Medical Sciences.

\section{Results}

The age mean \pm SD and man/woman percent of all patients who participated in our study were 59.28 \pm 10.29 and $84 / 16$ percent, respectively. The demographic data was summarized in Table 1. Statistically significant changes were observed in all patients who enrolled in the study. The employed paired T-test in both groups showed that primary PCI in both drug regime groups including Atorvastatin $80 \mathrm{mg} /$ day, before or after primary PCI, improved PTX3 and LVEF values. PTX3 and LVEF values in group with Atorvastatin pre-treatment changed after primary PCI, from $6.07 \pm 2.31 \mathrm{ng} / \mathrm{dL}$ to $4.92 \pm 1.98 \mathrm{ng} / \mathrm{dL}$ and $40.8 \pm 8.88$ to $45 \pm 6.77$, respectively. Both mentioned indexes in the Atorvastatin post-treatment group were also changed after Primary PCI, from 5.91 \pm $3.24 \mathrm{ng} / \mathrm{dL}$ to $5.37 \pm 3.6$ and $41.2 \pm 8.66$ to $44.9 \pm 7.79$, respectively. Also the vital capacity of the patients in two groups dramatically increased (Figure 1,2).

\begin{tabular}{|l|l|}
\hline Male/female (\%) & $84 / 16$ \\
\hline Age (year) & $59.28 \pm 10.29$ \\
\hline Smokers (\%) & $46 \%$ \\
\hline Triglyceride (mg/ L $\left.{ }^{-1}\right)$ & $154.6 \pm 82.05$ \\
\hline Diabetes Mellitus (\%) & $25 \%$ \\
\hline Hypertension (\%) & $27 \%$ \\
\hline High-density Lipoprotein cholesterol $\left(\mathrm{mg} / \mathrm{L}^{-1}\right)$ & $45.18 \pm 13.08$ \\
\hline Low- density Lipoprotein cholesterol $\left(\mathrm{mg} / \mathrm{L}^{-1}\right)$ & $104.18 \pm 33.16$ \\
\hline
\end{tabular}

Data are presented as number (\%) or mean

Table 1: Demographic data of the patients participated in this study 


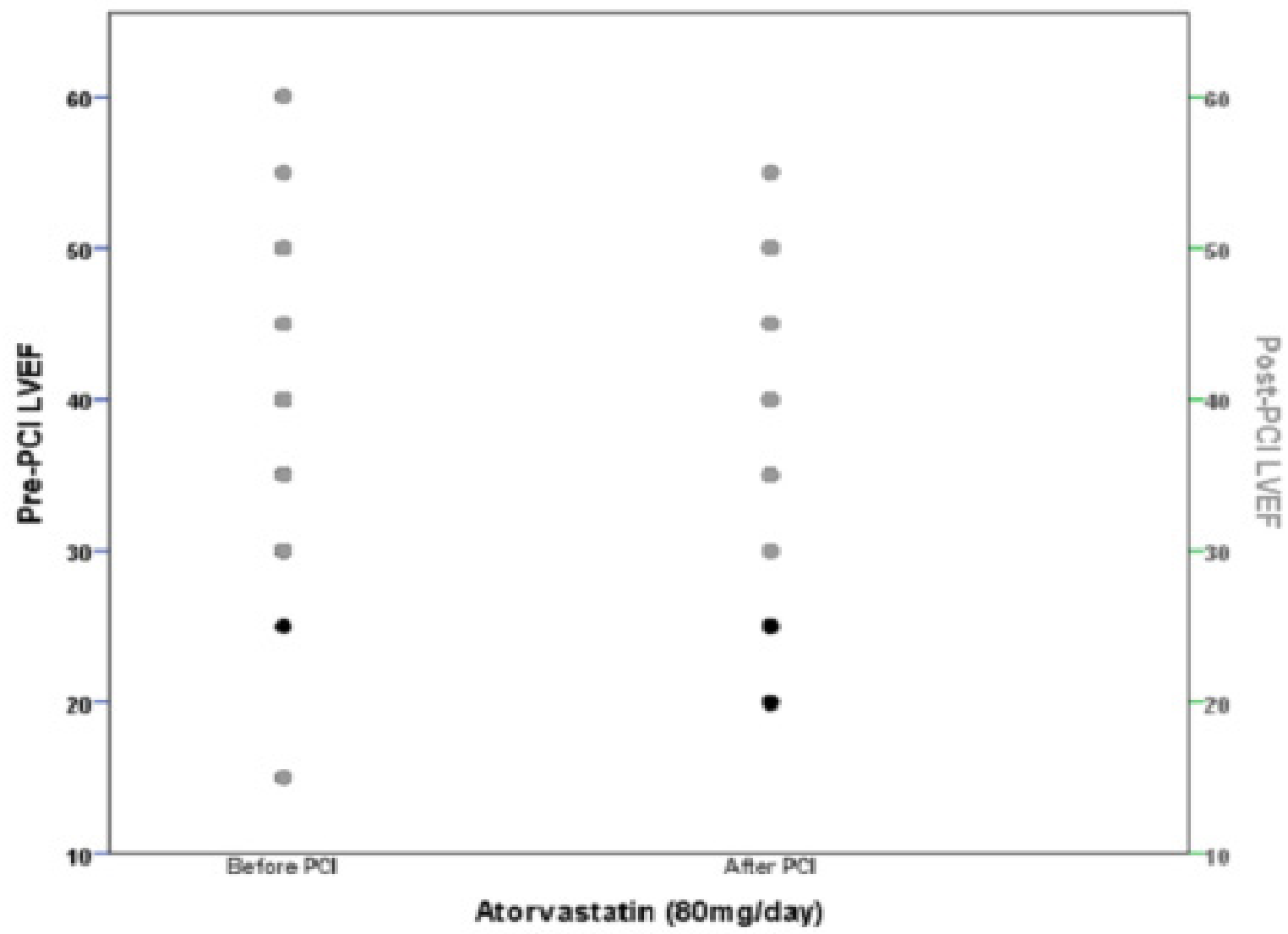

Figure 1: significant change of LVEF in both Atorvastatin pre and post -PCI treatment $(p=0.000)$ and statistically nonsignificant change of LVEF between two groups $(\mathrm{p}=0.946)$ at the end of study; LVEF: Left Ventricular Ejection Fraction, PCI: Percutaneous Coronary Intervention

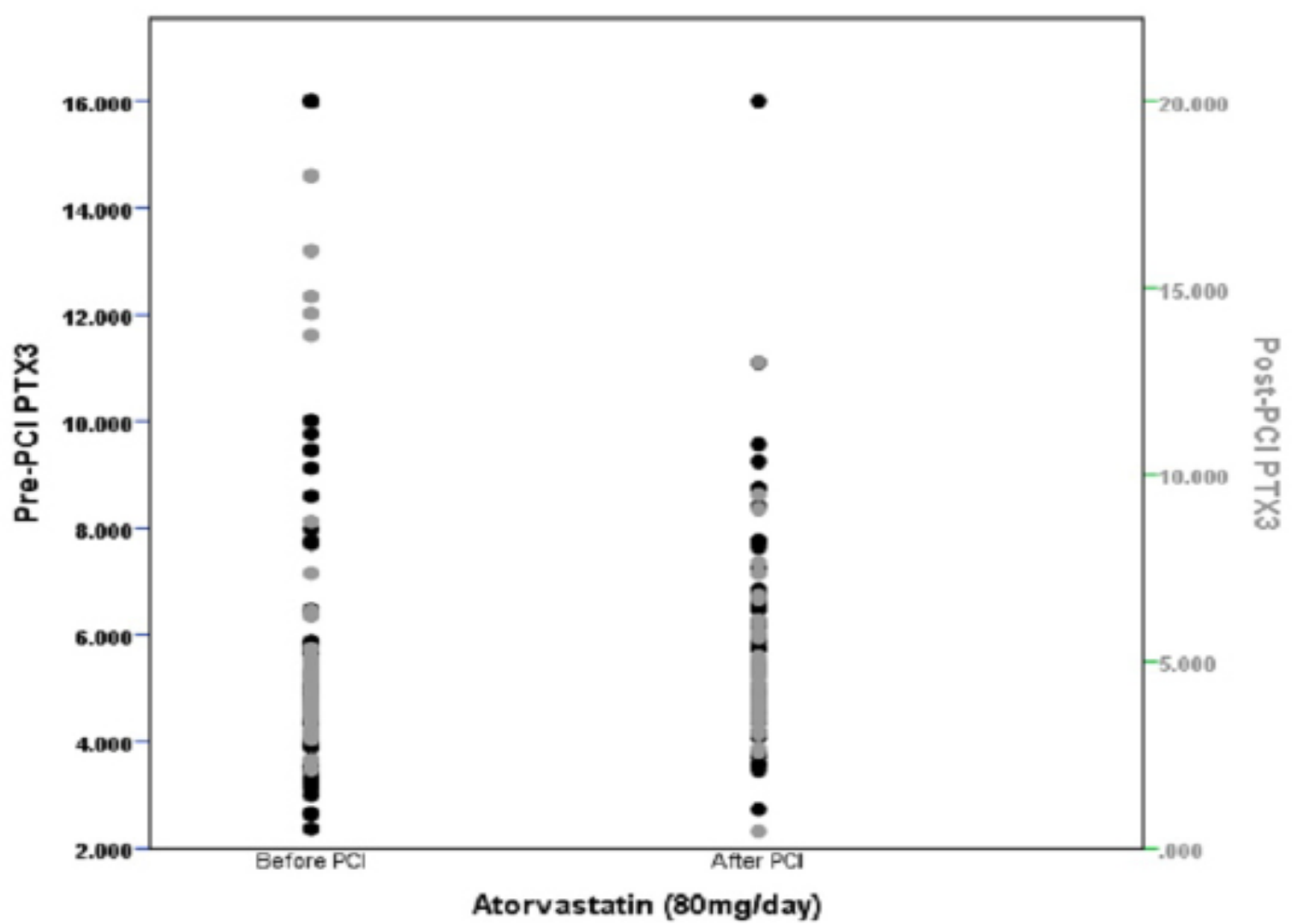

Figure 2: significant change of plasma PTX3 levels in both Atorvastatin pre and post-PCI treatment $(\mathrm{p}=0.043)$ and statistically non-significant change of PTX3 between two groups ( $\mathrm{p}=0.445)$ at the end of study; PTX3: Pentraxin 3, PCI: Percutaneous Coronary Intervention 
An Independent paired T-test showed that there is not statistically significant difference in the results of both LVEF $(\mathrm{P}$ value $=$ $0.946)$ and PTX3 (0.445) values in comparison between two groups.

\section{Discussion}

In this study, we showed that prescribing common dose of Aspirin and Clopidogrel before and high-dose of Atorvastatin (80 mg/ day) after primary PCI has the same results compared with conventional therapy including Atorvastatin pre-treatment beside Aspirin and Clopidogrel just before primary PCI.

In recent years, the numbers of studies that describe beneficial effects of statins on PCI outcome and lowering the blood lipid have dramatically increased. The beneficial effects of statins in heart failures and improving the life quality and quantity of patients with AMI have well known by several randomized studies [17-21]. Previous studies showed promising effects of Atorvastatin pre-treatment before PCI on expanding the lifetime as well as reducing perprocedural MI of patients during a long-term fallow up $[3,7,22]$. Lev and colleagues illustrated that statin pre-treatment effect on micro vascular functions and so, this event may improve coronary blood flow, after PCI [23]. Beneficial effects of statins are likely more than only lowering the blood lipid. As mentioned heretofore, statins can improve the endothelial function [24] and act as an anti- inflammatory agent [25,26], coronary microvascular dilator [27], as well as antithrombotic factor [28].

Several studies have demonstrated the beneficial effects of Atorvastatin pre-treatment before PCI but after the literature review, we could find just few studies about the efficacy of Atorvastatin post-treatment after PCI. Kuei and colleagues described that although Atorvastatin after PCI had a negative effect on Adiponectin system, anti-inflammatory and anti-atherogenic properties, but positive effects of Atorvastatin post- treatment after PCI on patients could frustrate the negative ones that mentioned [10]. Our compared results between two groups of our study is in agreement with mentioned study that showed the beneficial effects of Atorvastatin post-treatment, after a primary PCI.

In our study, LVEF as a vital capacity of the patients was significantly improved in both groups; from $40.8 \pm 8.88$ to $45 \pm 6.77$ in group with conventional drug regime and from $41.2 \pm 8.66$ to $44.9 \pm 7.79$ in the group with Atorvastatin post-treatment. The result of the study is in consistent with previous studies. Node et al showed that statin therapy for 3 months decreased left ventricular endsystolic volume in patients with idiopathic dilated cardiomyopathy [29]. In the other study, Teshima and colleagues demonstrated that Atorvastatin therapy early after an AMI onset improved LVEF [30]. Our study also showed that the results in both group are same. This finding illustrates that a single dose of Atorvastatin $(80 \mathrm{mg} /$ day $)$ after primary PCI can also have beneficial effect on cardiac functions just like the conventional drug plan.

PTX3 has known as a multifunctional conserved protein like C-Reaction Protein (CRP) that both of them are blood inflammatory indicators $[31,32]$. Although there are several studies that described the relationship between CRP, as an inflammatory indicator, and Atorvastatin [33-35], but we believe that the current study is one of the first human study that shows the effects of Atorvastatin therapy before and after primary PCI on PTX3. Our findings revealed that Atorvastatin treatment before or after primary PCI decreased the blood PTX3 values. As mentioned in the results, Atorvastatin pre and post-PCI treatment attenuated the plasma levels of PTX3. This finding may be related to anti-inflammatory effect of Atorvastatin that reduced the plasma levels of an inflammatory indicator, blood-circulating PTX3.

Our study had some limitations. The most important limitation of this study was enrolling patients in the study. Most of patients did not accept enrolling in our study, so samples achieving was a challenge. The authors believe that next studies on greater sample size will reveal results that are more interesting.

\section{Conclusion}

Our results indicate that Atorvastatin therapy after PCI, particularly in the emergency conditions that the patient is not stable and cannot take several pills, has the same beneficial effects on Cardiac functions compared with conventional drug regime before PCI.

\section{References}

1. Lim GB (2013) Global burden of cardiovascular disease. Nat Rev Cardiol 10: 59.

2. Nusca A, Melfi R, Patti G, Di Sciascio G (2010) Atorvastatin for reduction of myocardial damage during angioplasty trials. J Cardiovasc Med (Hagerstown) 11: 709-11.

3. Briguori C, Visconti G, Focaccio A, Golia B, Chieffo A, et al. (2009) Novel approaches for preventing or limiting events (Naples) II trial: impact of a single high loading dose of atorvastatin on periprocedural myocardial infarction. J Am Coll Cardiol 54: 2157-63.

4. Patti G, Pasceri V, Colonna G, Miglionico M, Fischetti D, et al. (2007) Atorvastatin pretreatment improves outcomes in patients with acute coronary syndromes undergoing early percutaneous coronary intervention: results of the ARMYDA-ACS randomized trial. J Am Coll Cardiol 49: 1272-8.

5. Kim JS, Kim J, Choi D, Lee CJ, Lee SH, et al. (2010) Efficacy of high-dose atorvastatin loading before primary percutaneous coronary intervention in ST-segment elevation myocardial infarction: the STATIN STEMI trial. JACC Cardiovasc Interv 3: 332-9.

6. Ricciardi MJ, Davidson CJ, Gubernikoff G, Beohar N, Eckman LJ, et al. (2003) Troponin I elevation and cardiac events after percutaneous coronary intervention. Am Heart J 145: 522-8. 
7. Di Sciascio G, Patti G, Pasceri V, Gaspardone A, Colonna G, et al. (2009) Efficacy of atorvastatin reload in patients on chronic statin therapy undergoing percutaneous coronary intervention: results of the ARMYDA-RECAPTURE (Atorvastatin for Reduction of Myocardial Damage During Angioplasty) Randomized Trial. J Am Coll Cardiol 54: 558-65.

8. Patti G, Chello M, Pasceri V, Colonna D, Nusca A, et al. (2006) Protection from procedural myocardial injury by atorvastatin is associated with lower levels of adhesion molecules after percutaneous coronary intervention: results from the ARMYDA-CAMs (Atorvastatin for Reduction of Myocardial Damage during Angioplasty-Cell Adhesion Molecules) substudy. J Am Coll Cardiol 48: 1560-6.

9. Sposito AC, Chapman MJ (2002) Statin therapy in acute coronary syndromes: mechanistic insight into clinical benefit. Arterioscler Thromb Vasc Biol 22: 152434.

10. Chan KC, Chou HH, Huang CN, Chou MC (2008) Atorvastatin administration after percutaneous coronary intervention in patients with coronary artery disease and normal lipid profiles: impact on plasma adiponectin level. Clin Cardiol 31: 253-8.

11. Rolph MS, Zimmer S, Bottazzi B, Garlanda C, Mantovani A, et al. (2002) Production of the long pentraxin PTX3 in advanced atherosclerotic plaques. Arterioscler Thromb Vasc Biol 22: e10-4.

12. Jenny NS, Arnold AM, Kuller LH, Tracy RP, Psaty BM (2009) Associations of pentraxin 3 with cardiovascular disease and all-cause death: the Cardiovascular Health Study. Arterioscler Thromb Vasc Biol 29: 594-9.

13. Peri G, Introna M, Corradi D, Iacuitti G, Signorini S, et al. (2000) PTX3, A prototypical long pentraxin, is an early indicator of acute myocardial infarction in humans. Circulation 102: 636-41.

14. Karakas MF, Buyukkaya E, Kurt M, Motor S, Akcay AB, et al. (2013) Serum pentraxin 3 levels are associated with the complexity and severity of coronary artery disease in patients with stable angina pectoris. J Investig Med 61: 278-85.

15. Namazi MH, Saadat H, Safi M, Vakili H, Alipourparsa S, et al. (2014) Pentraxin 3 Is Highly Specific for Predicting Anatomical Complexity of Coronary Artery Stenosis as Determined by the Synergy between Percutaneous Coronary Intervention with Taxus and Cardiac Surgery Score. Korean Circ J 44: $220-6$.

16. Haybar H, Assareh A, Ghotbi Y, Torabizadeh M, Bozorgmanesh M (2013) Incremental diagnostic value of circulating pentraxin in patients with intermediate risk of coronary artery disease. Heart 99: 640-8.

17. Arca M, Gaspardone A (2007) Atorvastatin efficacy in the primary and secondary prevention of cardiovascular events. Drugs 67 Suppl 1: $29-42$.

18. Austin PC, Mamdani MM (2005) Impact of the pravastatin or atorvastatin evaluation and infection therapy-thrombolysis in myocardial infarction 22/Reversal of Atherosclerosis with Aggressive Lipid Lowering trials on trends in intensive versus moderate statin therapy in Ontario, Canada. Circulation 112: 1296-300.

19. Cao S, Wang P, Cui K, Zhang L, Hou Y (2012) [Atorvastatin prevents contrast agent-induced renal injury in patients undergoing coronary angiography by inhibiting oxidative stress]. Nan Fang Yi Ke Da Xue Xue Bao 32: 1600-2.

20. Celik T, Kursaklioglu H, Iyisoy A, Kose S, Kilic S, et al. (2005) The effects of prior use of atorvastatin on coronary blood flow after primary percutaneous coronary intervention in patients presenting with acute myocardial infarction. Coron Artery Dis 16: 321-6.

21. Guay J, Ochroch EA (2014) Effects of adding statins before surgery on mortality and major morbidity: a meta-analysis. J Cardiothorac Vasc Anesth 28: 255-66. 22. Su Q, Li L, Liu Y, Zhou Y, Wang J, et al. (2014) Effect of intensive atorvastatin therapy on periprocedural PDCD4 expression in CD4+ T lymphocytes of patients with unstable angina undergoing percutaneous coronary intervention. Cardiology 127: 169-75.

23. Lev EI, Kornowski R, Vaknin-Assa H, Ben-Dor I, Brosh D, et al. (2009) Effect of previous treatment with statins on outcome of patients with ST-segment elevation myocardial infarction treated with primary percutaneous coronary intervention. Am J Cardiol 103: 165-9.

24. Hosokawa S, Hiasa Y, Tomokane T, Ogura R, Miyajima H, et al. (2006) The effects of atorvastatin on coronary endothelial function in patients with recent myocardial infarction. Clin Cardiol 29: 357-62.

25. Li Q, Deng SB, Xia S, Du JL, She Q (2013) Impact of intensive statin use on the level of inflammation and platelet activation in stable angina after percutaneous coronary intervention: a clinical study. Med Clin (Barc) 140: 532-6.

26. Macin SM, Perna ER, Farias EF, Franciosi V, Cialzeta JR, et al. (2005) Atorvastatin has an important acute anti-inflammatory effect in patients with acute coronary syndrome: results of a randomized, double-blind, placebo-controlled study. Am Heart J 149: 451-7.

27. Hinoi T, Matsuo S, Tadehara F, Tsujiyama S, Yamakido M (2005) Acute effect of atorvastatin on coronary circulation measured by transthoracic Doppler echocardiography in patients without coronary artery disease by angiography. Am J Cardiol 96: 89-91.

28. Violi F, Carnevale R, Pastori D, Pignatelli P (2014) Antioxidant and antiplatelet effects of atorvastatin by Nox2 inhibition. Trends Cardiovasc Med 24: 142-8.

29. Node K, Fujita M, Kitakaze M, Hori M, Liao JK (2003) Short-term statin therapy improves cardiac function and symptoms in patients with idiopathic dilated cardiomyopathy. Circulation 108: 839-43.

30. Teshima Y, Yufu K, Akioka H, Iwao T, Anan F, et al. (2009) Early atorvastatin therapy improves cardiac function in patients with acute myocardial infarction. J Cardiol 53: 58-64.

31. Mantovani A, Garlanda C, Bottazzi B, Peri G, Doni A, et al. (2006) The long pentraxin PTX3 in vascular pathology. Vascular Pharmacology 45: 326-30.

32. Du Clos TW (2013) Pentraxins: structure, function, and role in inflammation. Int Schol Res Notice Infla doi.org/10.1155/2013/379040.

33. Doggrell SA (2005) Relating statin therapy to C-reactive protein levels. Expert Opin Pharmacother 6: 1597-600.

34. Ridker PM, Cannon CP, Morrow D, Rifai N, Rose LM, et al. (2005) C-reactive protein levels and outcomes after statin therapy. N Engl J Med 352: 20-8.

35. Su JZ, Xue Y, Cai WQ, Huang QY, Chai DJ, et al. (2011) [Association between high sensitivity C-reactive protein and contrast induced acute kidney injury in patients with acute coronary syndrome undergoing percutaneous coronary intervention: impact of atorvastatin]. Zhonghua Xin Xue Guan Bing Za Zhi 39: 807-11. 


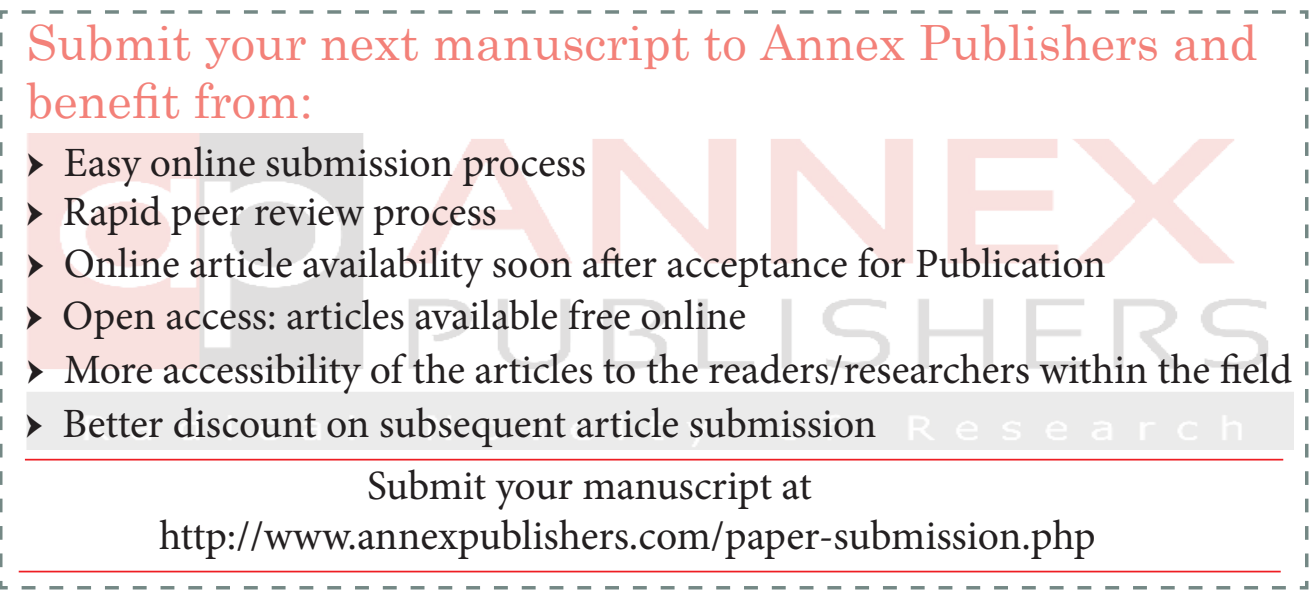

